\title{
Are smokers' self-reports of inhalation a useful measure of smoke exposure?
}

\author{
R STEPNEY \\ From the Department of Medicine, University of Cambridge Clinical School, Addenbrooke's Hospital, \\ Cambridge CB2 $2 Q Q, U K$
}

SUMMARY The relation between self-assessed and objective measures of inhalation was studied in 75 smokers who assigned themselves to one of four inhalation categories, and also estimated inhalation using a rating scale. The analysis of presmoking carbon monoxide concentration in expired air, and of the rise in carbon monoxide concentration over smoking, provided an objective measure of inhalation. There was a weak but significant correlation between self-rated inhalation and rise in carbon monoxide, but no correlation with the longer-term exposure measured by presmoking levels of carbon monoxide. Differences in exposure to carbon monoxide according to self-assessed inhalation category were non-significant. It is concluded that neither subjective measure of inhalation contributes usefully to the estimation of smoke exposure among smokers who inhale.

In population surveys smokers are routinely asked to estimate the extent of inhalation. ${ }^{1}$ Since the consequences to health of smoking depend on total exposure to toxic smoke components, the way in which cigarettes are smoked is as important a variable as the number and type of cigarette consumed. $^{2}{ }^{3}$ Epidemiological studies therefore also necessitate the collection of data on the presence or absence of inhalation, ${ }^{45}$ and its depth. ${ }^{6}$ Nevertheless, the associations that have been established between inhalation and the incidence of disease have been weak $^{6}$ or difficult to interpret, ${ }^{45}$ and the absence of a clear indication of the increased risk accompanying inhalation has been cited as evidence against the view that smoking causes disease. ${ }^{7}$ Although the apparent anomaly is probably due to the inaccuracy of smokers' self-reports about their inhaling habits, rather than to the absence of an association between inhalation and ill-health, the unreliability of traditional self-assessed inhalation measures needs to be confirmed. In the light of recommendations made by the International Union Against Cancer workshop on the measurement of smoking behaviour it is also of interest to assess the usefulness of possible alternative methods by which inhalation might be estimated. ${ }^{9}$

\section{Subjects and methods}

Seventy-five subjects ( 37 men; 38 women) took part in the study. All were regular smokers of between five and 50 king-size low, low-middle, or middle tar cigarettes a day. None smoked a pipe or cigars. Twenty-five were hospital or university employees. The remainder were recruited by personal contact or advertisement and represented a cross-section of the general cigarette-smoking population.

Each subject gave details of the type of cigarette smoked, consumption, the number of cigarettes smoked that day before coming to the laboratory, and the time since they had last smoked. They were then asked to place a cross anywhere along a single horizontal $100-\mathrm{mm}$ line to indicate how deeply they inhaled when smoking their usual brand of cigarette. One end of the line was labelled "not at all" the other "very deeply." They then chose from the categories "not at all/slightly/moderately/deeply" the alternative that best described the way they inhaled their usual brand.

A sample of exhaled air was obtained directly before and after subjects smoked a single cigarette of their own brand. To obtain the expired-air sample, subjects were asked to inhale, hold their breath for 20 seconds, and then exhale fully through a glass T-piece into two collection bags. The bag containing the first $500 \mathrm{ml}$ of exhalate was then discarded, and the carbon monoxide concentration of the remaining sample measured using a 2000 Series Ecolyzer. Carbon monoxide values obtained by this method correlate highly with blood carboxyhaemoglobin measures, are accurate, and have been recommended as an objective indication of the amount smokers 
inhale..$^{10} 12$ In our own laboratory and elsewhere $e^{11} 12$ the background level of carbon monoxide in non-smokers (due to environmental exposure and endogenous production of carbon monoxide) averages $5 \mathrm{ppm}$. All presmoking levels of carbon monoxide were therefore adjusted by the subtraction of $5 \mathrm{ppm}$ from the concentration observed.

In addition to any real variation in inhalation, presmoking levels of carbon monoxide are affected by the number of cigarettes smoked, the time since the last cigarette, and the carbon monoxide yield of the product smoked. Differences in standard yield also affect the rise in carbon monoxide over smoking. The potentially confounding effect of these variables was taken into account in two ways. Firstly, before investigating any differences in exposure between smokers who assigned themselves to the discrete inhalation categories, carbon monoxide values were adjusted to take into account the effect of yield and, where appropriate, number of cigarettes smoked and time since last smoking. This adjustment was accomplished by means of an analysis of covariance using the variables listed above. Secondly, in assessing the relationship between exposure to carbon monoxide and the smokers' scores on the continuous self-rating scale, multiple regression was used, enabling the variance due to yield, previous cigarette consumption, and time since last smoking to be extracted before the association between self-rating score and exposure to carbon monoxide was determined.

\section{Results}

INHALATION CATEGORIES

Among the present group of 75 smokers, only two claimed that they did not inhale. These two smokers had an average presmoking level of carbon monoxide of $11.3 \mathrm{ppm}$, and showed a rise over smoking of only $1.3 \mathrm{ppm}$. It is therefore likely that both were genuine non-inhalers. Nevertheless, no judgment can be made on the basis of these two subjects about the wider reliability of self-reported non-inhalation.

Mean concentration of carbon monoxide (ppm) in simokers judging themselves to be slight, moderate, and deep inhalers

\begin{tabular}{lllll}
\hline & $\begin{array}{l}\text { Inhale } \\
\text { slightly } \\
(n=4)\end{array}$ & $\begin{array}{l}\text { Inhale } \\
\text { moderately } \\
(n=46)\end{array}$ & $\begin{array}{l}\text { Inhale } \\
\text { deeply } \\
(n=23)\end{array}$ & $\begin{array}{l}\text { Significance } \\
\text { of difference }\end{array}$ \\
\hline $\begin{array}{l}\text { Presmoking* } \\
\text { Rise over } \\
\text { smoking }{ }^{\dagger}\end{array}$ & $(23.8)$ & 24.1 & 23.3 & NS \\
\hline
\end{tabular}

-Adjusted for $\mathrm{CO}$ yield of brand smoked, number of cigarettes smoked, and time since last smoking.

†Adjusted for $\mathrm{CO}$ yield.
The table shows the mean adjusted presmoking concentration of carbon monoxide, and rise in carbon monoxide over smoking, in subjects who assessed themselves as inhaling slightly, moderately, and deeply. The small number of smokers who considered themselves slight inhalers prevents the drawing of any conclusions about their exposure to carbon monoxide.

Presmoking levels of carbon monoxide were marginally lower in deep than in moderate inhalers, while the average rise in carbon monoxide over smoking was greater. In neither case, however, were the differences statistically significant.

INHALATION ASSESSED BY RATING SCALE

The mean rating-scale judgment made by smokers within each self-assessed inhalation category was appropriate to the category chosen. Nevertheless, smokers within each inhalation category varied considerably in their rating-scale assessment (fig). This suggested that the rating scale method might enable smokers to make a more sensitive and accurate estimate of the extent of inhalation. The relationships between rating-scale judgments and levels of carbon monoxide were therefore assessed by multiple regression. In this analysis the scores of all 75 subjects were used. After extracting variance due to yield, the number of cigarettes smoked, and time since last smoking, the rating scale estimate of inhalation correlated $r=0.09$ (NS) with presmoking level of carbon monoxide. After extracting variance due to yield, the rise in carbon monoxide over smoking correlated $r=0.29(p<0.05)$ with rating scale scores.

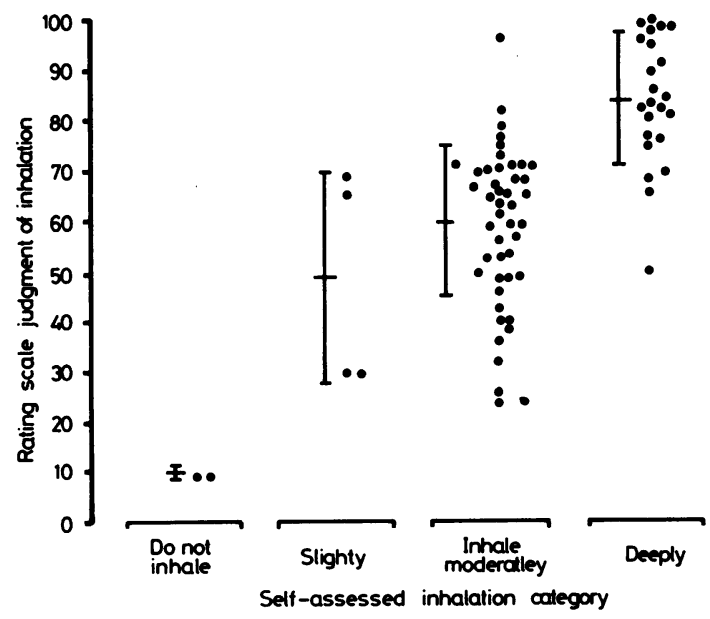

Rating scale judgments of inhalation related to self-assessed category of inhalation. Bars indicate mean $( \pm S D)$ rating scale scores in each group. 


\section{Discussion}

In a previous study of 500 male, middle-aged smokers belonging to a private health-insurance scheme, a statistically significant relationship was found between self-reported inhalation depth and a COHb-based measure of exposure to smoke. ${ }^{8}$ The differences according to inhalation category, however, were slight. The present study, by using a smaller but more representative group of smokers, confirms that for all practical purposes an inhaling smoker's self-report of inhalation depth is likely to be of little use in predicting actual exposure to toxic smoke components. In addition, this study suggests that any attempt to estimate inhalation by using a rating scale would not prove any more useful in assessing long-term exposure to smoke than the conventional method of asking smokers to assign themselves to an inhalation category.

The relationship between the carbon monoxide-based measures of inhalation and self-assessment is so poor that the validity of the carbon monoxide data might itself be called into question. The carbon monoxide measures were therefore correlated with three variables with which they have previously been found to be associated. Presmoking carbon monoxide concentration correlated $r=0.47(p<0.001)$ with the number of cigarettes smoked before attending the laboratory, and $r=-0.49)(p<0.001)$ with time since last smoking. The rise in carbon monoxide concentration over smoking correlated $r=0.36(p<0.01)$ with the standard carbon monoxide yield of the cigarette smoked. These correlations are similar to those previously reported. ${ }^{11}$

Given that expired-air carbon monoxide levels are a reliable and objective measure, the smoker's apparent inability to judge inhalation needs to be explained. Carbon monoxide is absorbed only when smoke is taken into the lungs, ${ }^{813}$ and probably duration as well as depth of inhalation are important in determining uptake. The present study followed previous practice in asking subjects to estimate depth of inhalation rather than duration. A previous small-scale study (15 subjects) showed that smokers' self-ratings of inhalation correlated reasonably well $(r=0.65)$ with mean inspired volume measured by chest pneumography. ${ }^{14}$ If the wider population of smokers is also able accurately to estimate the volume of inhaled smoke, the absence of a relationship between objective and conventional self-assessed measures of inhalation may be explained by the fact that the latter do not take account of the duration of inhalation.

It is also important to note that an individual smoker's intake of carbon monoxide will depend not only on the depth and duration of inhalation but also on the amount of carbon monoxide generated by that particular smoker's pattern of puffing on the cigarette. Thus two people smoking the same brand of cigarette and inhaling to the same extent will absorb very different quantities of carbon monoxide if one smoker has generated a great volume of smoke by taking frequent large puffs and the other has derived a small volume of smoke through taking infrequent small puffs. In the present study differences in carbon monoxide availability due to variations in standard carbon monoxide yield were taken into account, but differences due to the way a particular cigarette was smoked were not. It is now possible to measure and record in detail the puffing behaviour of individual smokers, and to duplicate their exact smoking pattern on a smoking machine. ${ }^{15}$ In this way the total volume of smoke taken, and the amount of carbon monoxide generated by a particular pattern of puffing (the carbon monoxide delivered "to the smoker's mouth") may be measured. In a preliminary study in this laboratory (40 subjects smoking their usual brand of middle or low-tar cigarette) the rise in expired-air carbon monoxide concentration over smoking was found to correlate $r=0.46(p<0.01)$ with the total volume of smoke taken from the cigarette and $r=0.56$ $(p<0.001)$ with the duplicated mouth-level delivery of carbon monoxide. If either or both of these variables were taken into account a closer relationship between self-assessed inhalation and intake of carbon monoxide might possibly emerge. Since, however, the use of such advanced measures on a large scale could probably not be considered, self-rated inhalation depth would even then remain of little practical use as a measure of smoke exposure.

I thank Peter Smith, of Cambridge University's department of community medicine, for his valuable advice on statistics, and the DHSS which provided figures for the standard carbon monoxide delivery of the different cigarette brands.

\section{References}

${ }^{1}$ Lee PN, ed. Statistics of smoking in the UK. London: Tobacco Research Council, 1976.

${ }^{2}$ Jarvis MJ, Russell MAH. Comment on the Hunter committee's second report. $\mathrm{Br}$ Med J 1980; 280: 994-5.

${ }^{3}$ Surgeon General. The changing cigarette. Washington: US Department of Health and Human Services, 1981.

4Schwartz D, Flamant R, Lellouch J, Denoix PF. Results of a French survey on the role of tobacco, particularly inhalation, in different cancer sites. J Natl Cancer Inst 1961; 26: 1085-1108. 
${ }^{5}$ Doll $\mathbf{R}$, Hill AB. Mortality in relation to smoking: ten years' observations of British doctors. $\mathrm{Br}$ Med J 1964; 1399-1410, 1460-7.

- Wynder EL, Stellman SD. Impact of long-term filter cigarette usage on lung and larynx cancer: a case control study. J Natl Cancer Inst 1979; 62: 471-7.

${ }^{7}$ Eysenck HJ. The causes and effects of smoking. London: Temple Smith, 1980.

${ }^{8}$ Wald N, Idle M, Bailey A. Carboxyhaemoglobin levels and inhaling habits in cigarette smokers. Thorax 1978; 33: 201-6.

${ }^{9}$ Gray N, Daube M. Guidelines for smoking control. 2nd ed. Geneva: International Union Against Cancer, 1980: appendix 2.

${ }^{10}$ Jarvis MJ, Russell MAH, Saloojee Y. Expired air carbon monoxide: a simple breath test of tobacco smoke intake. Br Med J 1980; 281: 484-5.

${ }^{11}$ Vogt TM, Selvin S, Widdowson G, Hulley SB. Expired air carbon monoxide and serum thiocyanate as objective measures of cigarette exposure. Am J Pub Health 1977; 67: 545-9.
${ }^{12}$ Wald NJ, Idle M, Boreham J, Bailey A. Carbon monoxide in breath in relation to smoking and carboxyhaemoglobin levels. Thorax 1981; 36: 366-9.

${ }^{13}$ Schoenfisch WH, Hoop KA, Streulens BS. Carbon monoxide absorption through the oral and nasal mucosa of cynomolgus monkeys. Arch Environ Health 1980; 35: $152-4$.

${ }^{14}$ Rawbone RG, Murphy K, Tate ME, Kane SJ. The analysis of smoking parameters: inhalation and absorption of tobacco smoke in studies of human smoking behaviour. In: Thornton RE, ed. Smoking behaviour: physiological and psychological influences. Edinburgh: Churchill Livingstone, 1978.

${ }^{15}$ Creighton DE, Noble MJ, Whewell RT. A portable smoking pattern recorder. Biotelemetry and Patient Monitoring 1979; 6: 186-91.

CORRECTIONS

Assessment of efiiciency in potential screening in Wilson's disease (December 1981)

On page 276 , line 16 on right, the figure " $8 / 116$ " should be " $108 / 116$ " as in table 3 .
Limitations of case-control studies in the detection of environmental carcinogens (December 1981)

On page 287 the following equation should read

$N=\left\{z_{\alpha} \sqrt{(1+1 / a) p_{q}}+Z_{\beta} \sqrt{p_{1}\left(1-p_{1}\right)+p_{2}\left(1-p_{2}\right)} / a\right\}^{2} /\left(p_{1}-p_{2}\right)^{2}$ 